\title{
Well-Packed Chains and Aggregates in the Emission Mechanism of Conjugated Polymers
}

Kang-Yung Peng and Show-An Chen*

Department of Chemical Engineering, National Tsing-Hua University, Hsinchu, 30013 Taiwan, R.O.C.

Wun-Shain Fann

Institute of Atomic and Molecular Sciences, Academia Sinica, P.O. Box 23-166, Taipei, 10617 Taiwan, R.O.C.

Su-Hua Chen and An-Chung Su

Institute of Materials Science and Engineering, National Sun Yat-sen University, Kaohsiung 804, Taiwan, R.O.C.

* Corresponding author E-mail: sachen@che.nthu.edu.tw

\section{Instrumentation}

Molecular weights of the polymers were measured by gel permeation chromatography (GPC) against polystyrene standards using a liquid chromatography (Waters PreLC 4000 system) with 3 columns (Styragel® HR 2, 3 and 4) in series and a UV-Vis absorbance detector (Waters 2487). The carrier solvent used was degassed tetrahydrofuran. 
TGA measurement was carried out under a dry nitrogen atmosphere using a thermogravimetric analyzer (DuPont model 2950) from room temperature to $800 \square$ at a heating rate of $25 \square / \mathrm{min}$. All samples were preheated at $100 \square$ for 30 minutes under nitrogen to remove any trace of solvent. Thermal weight loss peak $\left(\mathrm{T}_{\mathrm{w} . l . p .}\right)$ was taken as the temperature at maximum derivative of percentage weight loss with respect to temperature.

Ultraviolet-Visible (UV-Vis) spectra were recorded using a UV-Vis spectrometer (Perkin-Elmer model lambda 19). Steady-state continuous wave (CW) photoluminescence (PL) and excitation (PLE) spectra were recorded using a luminescence spectrometer (Aminco-Bowman Series 2).

The time-correlated single photon counting (TCSPC) technique was used to measure luminescence decay curves. A 4096-channel TCSPC system was set up with a microchannel plate photomultiplier tube (Hamamatsu Photonics R3809U-50) and a spectrometer (Edinburgh, Lifespec-ps and TCC900 data acquisition card). The instrument response function (IRF) was measured on a thick parafilm film, by which the excitation light can be scattered, and a 45-ps full width at mid height (FWMH) was obtained. Optics was carefully aligned so that the instrument response is wavelength-independent. (This was verified by monitoring IRF of the 395-nm excitation and then its 790-nm second harmonic.) The excitation source in the TCSPC experiments was provided by the frequency-doubled output (second harmonic generator, SHG: Inrad 5-050) of a mode-locked 76-MHz Ti-Sapphire laser (Coherent Mira900) pumped by a 10-W diode laser (Coherent Verdi-V10). The FWMH of the Ti-sapphire laser is about 130 fs. The rate of excitation was reduced to $4.75 \mathrm{MHz}$ by a pulse picker (Coherent Mira 9200) between Ti-Sapphire laser and SHG to prevent cross contamination of signals between the adjacent excitations. The peak counts of all the luminescence decay curves were accumulated to 10,000 . Measurements on solution samples were performed immediately after throughout stirring the solutions. Most optical spectra of polymer solutions presented in this work were measured using a 1-cmpathlength cuvette but for concentrated solutions their absorption measurements were recorded from a 0.1-cm-pathlength cuvette or a very thin liquid layer confined between two slide glasses in contact. 


\section{Synthesis and characterizations of Monomers and Polymers}

The synthesis of the monomers and polymers are outlined below in Scheme 1S.

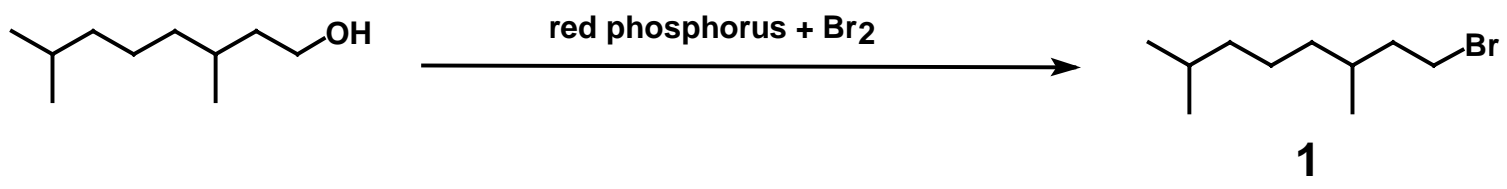

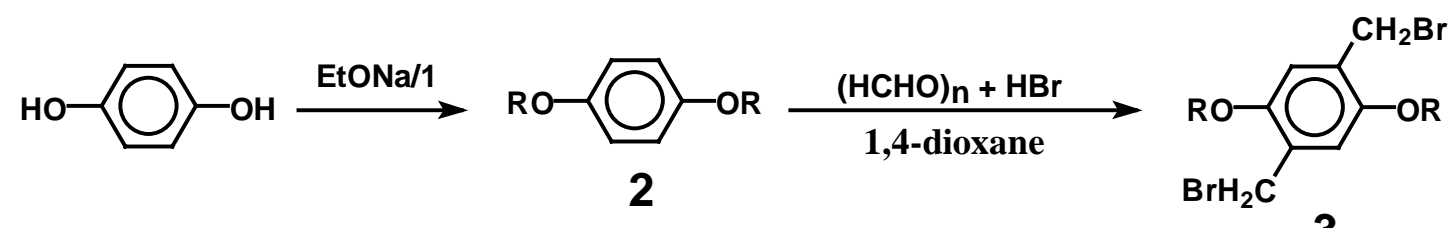<smiles>COc1ccc(OCC(=O)OCC(=O)OCc2ccccc2)cc1</smiles><smiles>COc1cc(CBr)c(OC)cc1CBr</smiles>

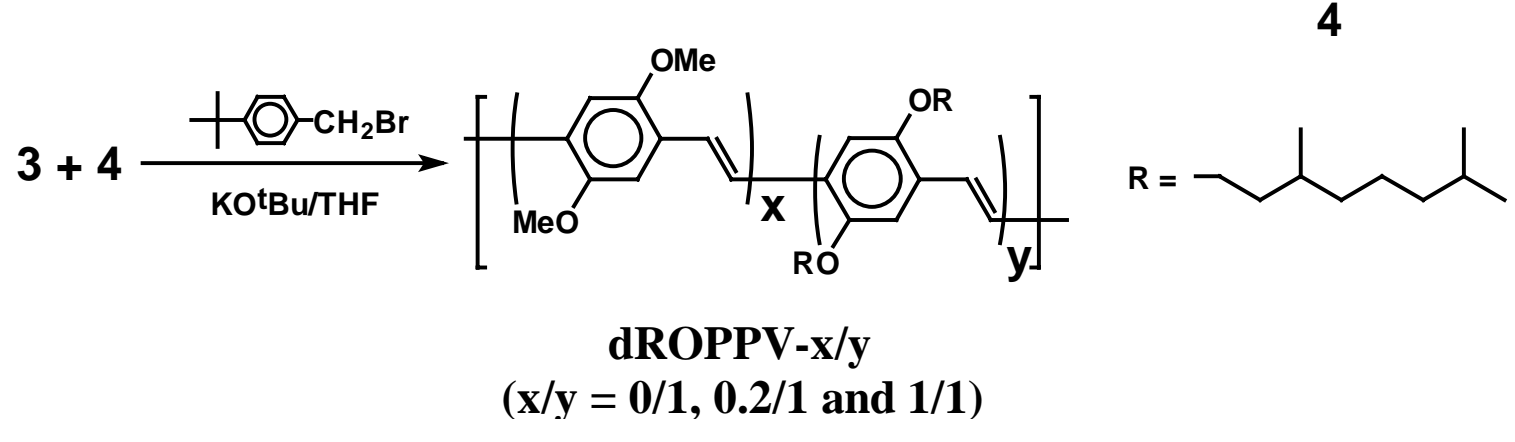

Scheme 1S. Synthesis route of monomers and the dROPPV-x/y copolymers. 


\section{2-1 preparation of Monomers}

3,7-dimethyloctyl bromide (1) Into a mixture of 3,7-dimethyloctyl alcohol (420 g, $2.65 \mathrm{~mol})$ and red phosphorus $(27.8 \mathrm{~g}, 0.897 \mathrm{~g})$ at $75 \square$, bromine $(303 \mathrm{~g}, 1.89 \mathrm{~mol})$ was added dropwisely. The dropping rate was so slow that it took about 6 hours to complete the addition. After further refluxing at $75 \square$ for 24 hours, the reaction was cooled to the room temperature and $100 \mathrm{~mL}$ water was then carefully dropped in. The solution was extracted by ethyl ether and $1 \mathrm{~N} \mathrm{NaOH}_{(\mathrm{aq})}$. Concentrating the ether layer and distilling at $90 \square$ in vacuum afforded 3,7-dimethyloctyl bromide (1) (496 g). The yield was $85 \% .{ }^{1} \mathrm{H}$ NMR $\left(500 \mathrm{MHz}, \mathrm{CDCl}_{3}\right): \delta 3.40(2 \mathrm{H}, \mathrm{m}), 1.85(1 \mathrm{H}, \mathrm{m}), 1.62(2 \mathrm{H}, \mathrm{m}), 1.52(1 \mathrm{H}, \mathrm{m}), 1.28$ $(3 \mathrm{H}, \mathrm{m}), 1.15(3 \mathrm{H}, \mathrm{m}), 0.90(9 \mathrm{H}, \mathrm{m})$.

1,4-bis(3',7'-dimethyloctyloxy)-benzene (2) Sodium cubes (28 g, $1.2 \mathrm{~mol})$ was added into $400 \mathrm{~mL}$ 99.5\% ethanol under nitrogen atmosphere in an ice bath. After the refluxing stopped, hydroquinone (55 g, $0.5 \mathrm{~mol})$ was added and the reaction was kept at $80 \square$ for one hour. Ethanol $100 \mathrm{~mL}$ and (1) (240 g, $1.1 \mathrm{~mol}$ ) was then added. After refluxing at $80 \square$ for 4 days, the reaction was cooled to the room temperature. The solution was firstly concentrated and then extracted by ethyl ether and water. Concentrating the ether layer and distilling at $240 \square$ in vacuum afforded 1,4-bis(3', 7'dimethyloctyloxy)-benzene (2) (183 g). The yield was $94 \% .{ }^{1} \mathrm{H} \mathrm{NMR}\left(500 \mathrm{MHz}, \mathrm{CDCl}_{3}\right): \delta 6.81$ (4H,s), $3.93(4 \mathrm{H}, \mathrm{m}), 1.76(2 \mathrm{H}, \mathrm{m}), 1.65(4 \mathrm{H}, \mathrm{m}), 1.55(2 \mathrm{H}, \mathrm{m}), 1.33(6 \mathrm{H}, \mathrm{m}), 1.15(6 \mathrm{H}, \mathrm{m}), 0.93-0.86$ $(18 \mathrm{H}, \mathrm{d}$ of $\mathrm{d})$.

\section{2,5-dibromomethyl-1,4-bis(3',7'-dimethyloctyloxy)-benzene (3) 1,4-bis(3',7'- dimethyloctyloxy)-}

benzene (2) (30 g, $0.078 \mathrm{~mol})$, paraformaldehyde (15g, effectively as $0.5 \mathrm{~mol}$ formaldehyde), $100 \mathrm{~mL}$ hydrobromic acid and $100 \mathrm{~mL} 33$ w.t.\% $\mathrm{HBr}$ in HOAc was added to $200 \mathrm{ml}$ 1,4-dioxane. The reaction was refluxed at $80 \square$ for 24 hours. After cooling down to the room temperature, a solid floating over the solution appeared and was collected. The solid was firstly dissolved in ether and then washed with water. Concentrating the ether layer afforded the coarse product. 2,5-dibromomethyl-1,4-bis(3', 7'dimethyloctyloxy)-benzene (3) (23 g) was obtained by the recrystallization from its solution in 150 
mL hexane. The yield was $52 \% .{ }^{1} \mathrm{H}$ NMR (500 MHz, $\left.\mathrm{CDCl}_{3}\right): \delta 6.84(2 \mathrm{H}, \mathrm{s}), 4.50(4 \mathrm{H}, \mathrm{S}), 3.99$ (4H,m), $1.83(2 \mathrm{H}, \mathrm{m}), 1.65(4 \mathrm{H}, \mathrm{m}), 1.55(2 \mathrm{H}, \mathrm{m}), 1.33(6 \mathrm{H}, \mathrm{m}), 1.15(6 \mathrm{H}, \mathrm{m}), 0.93-0.86(18 \mathrm{H}, \mathrm{d}$ of d). The bromomethylation can evolve carcinogenic bromomethyl ethers and must be conducted in an efficient cupboard with a great caution.

2,5-dibromomethyl-1,4-dimethoxy-benzene (4) 1,4-dimethoxybenzene (1) (55.3 g, $0.4 \mathrm{~mol})$, paraformaldehyde (30g, effectively as $1.0 \mathrm{~mol}$ formaldehyde) and $200 \mathrm{~mL} 33$ w.t.\% $\mathrm{HBr}$ in HOAc was added in to $350 \mathrm{ml}$ galactic acetic acid. The reaction was refluxed at $80 \square$ for 24 hours. After cooling down to the room temperature, the solid precipitated from the solution was collected and then washed by methanol. Recrystallization from $2 \mathrm{~L}$ chloroform afforded $67 \mathrm{~g}$ (1) (0.21 mol). The yield was $52 \%$. ${ }^{1} \mathrm{H}$ NMR (500 MHz, $\left.\mathrm{CDCl}_{3}\right): \delta 6.85$ (2H, s), 4.51 (4H, s), 3.85 (6H, s).

\section{2-2 Polymerization}

The solvent used for polymerization was anhydrous and a dry nitrogen atmosphere was adopted. The nomenclature of the copolymer dROPPV-1/1 is based on the molar feeding ratio (and also the real composition, as revealed by TGA) of the comonomers, (4)/(3). For polymer dROPPV-0/1, only monomer (3) was polymerized.

dROPPV-1/1 Monomer (3) (1.44 g, $0.0025 \mathrm{~mol})$, (4) (0.81 g, $0.0025 \mathrm{~mol})$ and tert-butyl benzyl bromide $1 \mathrm{~mL}(1.2 \mathrm{~g}, 0.005 \mathrm{~mol})$ was added to $45 \mathrm{~mL}$ anhydrous tetrahydrofuran (THF) under a nitrogen atmosphere. Into this mixture a solution of potassium tert-butoxide in THF $(3.2 \mathrm{~g}$ in $100 \mathrm{~mL}$, $0.28 \mathrm{M}$ ) was added dropwisely. After reacting at the room temperature for 24 hours, $200 \mathrm{~mL}$ methanol was added and the red precipitate was collected. Dissolving it in $300 \mathrm{~mL}$ chloroform and precipitating by $300 \mathrm{~mL}$ methanol afforded polymer powder. The weight of the product after drying in vacuum was $0.91 \mathrm{~g}$ and the yield is $63 \%$. Weight-averaged molecular weight $\left(\mathrm{M}_{\mathrm{w}}\right)=560,000$. Polydispersity index (PDI, $\left.\mathrm{M}_{\mathrm{w}} / \mathrm{M}_{\mathrm{n}}\right)=2.5$, Number-averaged degree of polymerization $\left(\mathrm{P}_{\mathrm{n}}\right)=778$. (The real composition of polymer was verified to be the same as the molecular ratio of feeding. The analysis is described in the 
next section, 2-3. Therefore the average molecular weight of repeating units and then Pn can be calculated.)

dROPPV-0.2/1 (3) (2.88 g, $0.005 \mathrm{~mol})$, (4) (0.32 g, $0.001 \mathrm{~mol})$ and tert-butyl benzyl bromide $1.2 \mathrm{~mL}$ $(1.4 \mathrm{~g}, 0.006 \mathrm{~mol})$ was added to $55 \mathrm{~mL}$ anhydrous tetrahydrofuran (THF) under a nitrogen atmosphere. Into this mixture a solution of potassium tert-butoxide in THF $(3.8 \mathrm{~g}$ in $120 \mathrm{~mL}, 0.28 \mathrm{M})$ was added dropwisely. The remaining procedures were the same as for dROPPV-1/1. The final product weighted $1.74 \mathrm{~g}$ and the yield was $78 \% . \mathrm{M}_{\mathrm{w}}: 621,000$. PDI: 2.3 .

dROPPV-0/1 The conditions of polymerization were the same as those in the dROPPV-1/1, except that only the monomer $(3)(2.88 \mathrm{~g}, 0.005 \mathrm{~mol})$ was used for the polymerization. The final product weighted $1.44 \mathrm{~g}$ and the yield was $70 \% . \mathrm{M}_{\mathrm{w}}=805,000 . \mathrm{PDI}=3.8 . \mathrm{P}_{\mathrm{n}}=512$.

\section{2-3 Quantification of the Polymer Composition}

In the thermogravimetric analysis (TGA) of poly[2,5-dialkoxy-1,4-phenylene vinylene) (dROPPV$\mathrm{x} / \mathrm{y}$ ), the weight loss before the degradation of the polymer main chains is caused by the loss of alkoxy side chains. (Ref.: Kuen-Ru Chuang, "Studies on Sructure/Properties of Poly(p-phenylene vinylene)s and Their Application in Light-Emitting Diode", PhD. Thesis, National Tsing Hua University, Taiwan, 1996/12.). Therefore, the relative amount of the methoxy and 3,7-dimethyloctyloxy side chains (i.e., the real composition) can be evaluated from TGA.

Figure $1 \mathrm{~S}$ shows the TGA results for dROPPV-x/y copolymers. The polymers start to lose their weight at about $390 \sim 400 \square$. Both the actual and the theoretic values of the weight loss percentages after a complete loss of alkyloxy side groups are listed in Table 1S. A close match between these two manifests that the comonomers are of equal reactivity toward the polymerization and that the actual composition of the copolymers was the same as the feed ratio of comonomers. 

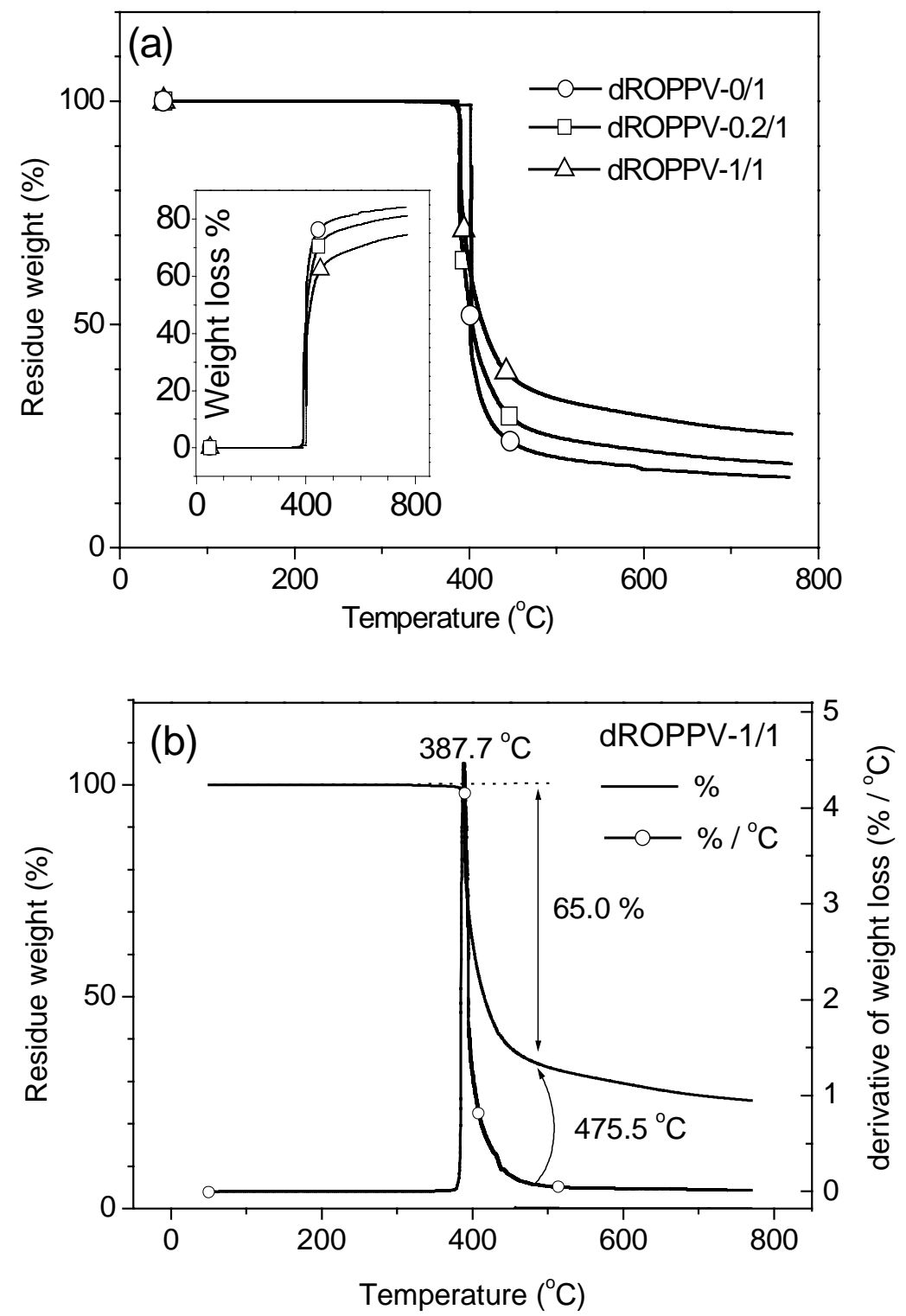

Figure 1S. (a) Thermogravimetric curves of dROPPV-x/y copolymers. The insert shows the corresponding curves for weight losses at different temperatures. (b) TGA and DTA (derivative thermogravimetric analysis) curves of $\mathrm{dROPPV}-1 / 1$ illustrating the characteristic values, $\mathrm{T}_{\mathrm{w} .1 . \mathrm{p} .}$, weight loss percentage and weight loss temperature, as defined below in the notes of Table 1S. 
Table 1S. Characteristic values of dROPPV-x/y copolymers in the thermogravimetric analysis (TGA).

\begin{tabular}{lcccc}
\hline Polymer & $\begin{array}{c}\mathrm{T}_{\text {w.l.p. }} \\
\square \square \square\end{array}$ & $\begin{array}{c}\text { Temperature for complete } \\
\text { weight loss of side chains } \\
\text { b) } \\
\square \square\end{array}$ & $\begin{array}{c}\text { Experimental } \\
\text { weight loss } \\
\text { percentage }\end{array}$ & $\begin{array}{c}\text { Theoretic } \\
\text { weight loss } \\
\text { percentage }^{\text {c) }}\end{array}$ \\
\hline dROPPV-1/1 & $\mathbf{3 8 7 . 7}$ & $\mathbf{4 7 5 . 5}$ & $\mathbf{6 5 . 0}$ & $\mathbf{6 5 . 3}$ \\
\hline dROPPV-0.2/1 & $\mathbf{3 8 4 . 6}$ & $\mathbf{4 7 1 . 0}$ & $\mathbf{7 3 . 6}$ & $\mathbf{7 2 . 5}$ \\
\hline dROPPV-0/1 & $\mathbf{3 9 8 . 1}$ & $\mathbf{4 5 1 . 2}$ & $\mathbf{7 6 . 6}$ & $\mathbf{7 5 . 9}$ \\
\hline
\end{tabular}

a) $T_{\text {w.l.p. }}$ (weight-loss peak temperature): the temperature at which the first derivative of weight loss with respect to temperature reaches the maximal value.

b) Based on the actual complete loss of alkyloxy side groups at which the first derivative of weight loss percentage to with respect temperature reaches constant.

c) Based on the complete loss of all methoxy and 3,7-dimethyloctyloxy side groups, as calculated from the feeding ratio of comonoers $(x / y)$. Theoretical $\%=[62 x+314 y] /[162 x+414 y]$. For dROPPV $-1 / 1$, $\mathrm{x}=\mathrm{y}=1$. 